\title{
Novel and Conventional Preclinical Models to Investigate Bone Metastasis
}

\author{
Tiina E. Kähkönen ${ }^{1}$ • Jenni Bernoulli ${ }^{1}$ • Jussi M. Halleen ${ }^{1}$ • Mari I. Suominen ${ }^{1}$
}

Published online: 16 January 2019

(C) The Author(s) 2019

\begin{abstract}
Purpose of Review The purpose of this review is to emphasize the use of bone metastasis models in preclinical research. As classical models have been thoroughly discussed in recent reviews, we here highlight the most important aspects from these papers with a special focus on novel models developed during the past 5 years.

Recent Findings Preclinical mouse models to study bone metastasis can be divided by cancer cell inoculation techniques (spontaneous, systemic, or local) or by immunological background of the mice (immunodeficient, syngeneic, or humanized). Additionally, novel computational, in vitro co-culture, and humanized bone models have been established.

Summary Various models can be used to approach distinct research questions. Understanding limitations of the models is essential when planning a study and interpreting the results. Development of novel models will increase understanding of the complex biology and advance the discovery of new therapies targeting bone metastases.
\end{abstract}

Keywords Bone metastases $\cdot$ Preclinical bone metastasis model $\cdot$ Systemic model $\cdot$ Intratibial model $\cdot$ Humanized models

\section{Introduction}

Bone metastases are a major cause of morbidity and mortality in cancer patients [1-3]. Seventy to eighty percent of advanced breast and prostate cancer patients develop bone metastases, and metastases are also very common in lung, kidney, and thyroid cancers and in melanoma $[2,3 \cdot, 4]$. Thus, bone metastases are a major influencer in the natural history of these cancers. Bone metastases cause severe pain and increase the risk of fractures leading to decreased quality of life $[2,4]$. The median survival time after diagnosis of bone metastases is only 2-3 years [2] and therefore, there is a great need to develop cancer therapies targeting bone metastatic disease.

Formation of bone metastases is a complex process. At very early stages, tumor cells influence the bone microenvironment through secretion of cytokines and exosomes that can

This article is part of the Topical Collection on Molecular Biology of Bone Metastasis

Mari I. Suominen

mari.suominen@pharmatest.com

1 Pharmatest Services, Itäinen Pitkäkatu 4C, 20520 Turku, Finland increase bone turnover and create a pre-metastatic niche [5•, 6]. The cytokines increasing bone resorption include for example interleukin (IL)-8 and hepatocyte growth factor, which are released at the site of the primary tumor by heparanase action [7]. Exosomes can increase vascular leakiness at the pre-metastatic site and educate the bone marrow progenitors via the MET receptor [8]. Exosome-like vesicles carrying miR-192 have also been noted to alter bone colonization [9]. Furthermore, signals from the primary tumor recruit monocytes, mesenchymal stem cells, and immature myeloid cells from the bone marrow to the primary tumor, where they are educated to become tumor-associated macrophages (TAMs), cancer-associated fibroblasts (CAFs), and myeloid-derived suppressor cells (MDSCs), supporting the metastatic process $[10 \bullet, 11]$. The following steps have been thoroughly described by Johnson and Suva in their review "Hallmarks of bone metastasis" [1]. These hallmarks are extravasation, disruption of bone homeostasis, secretion of osteolytic growth factors, engagement in bone niche, interactions with the bone marrow microenvironment of the host, and responses to the physical environment, which are all needed in order to develop clinically detectable bone metastases [1].

Bone is a favorable microenvironment for metastasis. It contains many growth factors such as parathyroid hormone- 
related peptide (PTHrP), transforming growth factor beta (TGF- $\beta$ ), and many cytokines including IL-6, IL-8, and tumor necrosis factor alpha (TNF- $\alpha)$, which support the early homing of cancer cells to bone as well as tumor growth [5•]. The term vicious cycle refers to increased bone destruction caused by the cancer cells, which in turn supports cancer cell growth $[3 \bullet, 5 \bullet]$. Shortly, it describes the "self-feeding" process which originated from the discovery of PTHrP as a major regulator of growth of bone metastases, and the effects of which are caused by increased differentiation of bone-forming osteoblasts. Increased osteoblast differentiation and secretion of receptor activator of nuclear factor kappa-B ligand (RANKL) from osteoblasts leads to increased activity of bone-resorbing osteoclasts and increased bone resorption. Many growth factors and hormones are bound to the bone matrix upon mineralization, and once released they promote cancer growth in bone. The vicious cycle is one of the reasons that make the tumor microenvironment in bone unique and difficult to modulate by therapies. The vicious cycle is recapitulated in in vivo models, but modeling it in vitro has proven to be very challenging. However, an in vitro model by Krishnan and colleagues models the vicious cycle in silico and allows the study of osteobiological and osteopathological processes and effects of potential therapeutics [12].

Bone metastases can be divided into two categories, osteolytic and osteoblastic [3•, 4]. Increased bone resorption is observed in osteolytic lesions, and they are more common for example in breast and lung cancers [4]. Increased bone formation is more profound in osteoblastic lesions, and they are more common in prostate cancer [4]. Despite the classification based on the dominant process, usually both bone formation and resorption occur simultaneously. This is demonstrated by the fact that in prostate cancer patients with osteoblastic lesions, resorption markers can be even more elevated than in patients with osteolytic lesions [13]. In preclinical models, tumor growth in bone and tumor-induced bone lesions can be imaged with conventional imaging modalities, such as X-ray, micro-computed tomography ( $\mu \mathrm{CT})$, bioluminescence imaging (BLI), magnetic resonance imaging (MRI), or positron emission tomography (PET/SPECT) [3•]. During recent years, more attention has been paid to imaging rare events such as tumor cell dissemination or dormancy with more advanced techniques. For example, real-time in vivo imaging of bone metastatic tumors by near-infrared techniques could be useful in more precise tumor detection in bone, and for detecting small tumors [14]. More precise tumor detection can also be achieved by intravital imaging of bone marrow and tumor cells by two-photon microscopy [15•]. Additionally, bone biomarkers can be used to study the changes in bone turnover in preclinical and clinical settings [16, 17]. When tested in tumor models which cause osteolytic and osteoblastic lesions in vivo, the bone formation markers $\mathrm{N}$ terminal propeptide of type I collagen (PINP) and osteocalcin
(OC) and the resorption markers C-terminal telopeptide of type I collagen (CTX-I) and tartrate-resistant acid phosphatase 5b (TRACP 5b) were increased at early stages and were downregulated by the treatment of zoledronic acid [17]. At later stages, the levels were stabilized in an osteoblastic model and could not be inhibited by zoledronic acid [17]. OC was the best marker to correlate with tumor burden measured by BLI [17]. However, when considering correlation, one must distinguish bone formation and tumor growth. If bone formation is very strong, a smaller proportion of the lesion consists of cancer cells and a larger proportion consists of bone, and thus the correlation may be even negative. Measuring and understanding bone turnover through biomarkers has been essential in developing bone-targeted therapies against bone metastases [16]. In clinical trials, bone biomarkers have a key role in deciding whether a new bone-targeted agent will continue in clinical development [16]. In preclinical studies, the use of biomarkers is not compulsory but their use can increase clinical predictivity [16].

One of the most important obstacles in developing good compounds against bone metastatic disease has been the misuse of in vivo models. The most commonly used models in oncology are subcutaneous models. They are also used to answer questions of efficacy against metastatic cancers. Especially in the case of bone metastases, the biology and cancer-induced changes are results of a complex activation of cascades of operations that can be modeled only in the correct bone metastatic microenvironment. The placement of cancer cells to a microenvironment highly different from their origin rarely results in preclinical findings that correspond to clinical efficacy [18•].

Different models that can be used to study bone metastasis are extensively described by Jinnah and colleagues [2]. Additionally, Simmons and colleagues explain in detail the models both by cell type and by inoculation route, as well as other approaches like inducible models [3•]. In the context of bone metastases, transgenic mouse models are challenging because bone metastases are generally not observed even in breast or prostate cancer models $[3 \bullet, 19]$. The few exceptions with occasional bone metastases include the TRAMP (transgenic adenocarcinoma mouse prostate) x FBV model [20], the Cryptdin-2-Tag prostate cancer model, and the Wcre;Cdh1 $1^{F}$;Trp $53^{F}$ breast cancer model [19]. Each model has advantages and disadvantages, and understanding the possibilities and limitations is crucial in selecting the most suitable model for a study [3•]. Furthermore, characterization of these models and development of new models are essential for better understanding of the mechanisms for development and growth of bone metastases, and for identifying key targets for novel therapeutics [3•]. When developing new models, major focus should be paid to clinical relevance [3•]. In the following chapters, we highlight the most recent work in preclinical models of bone metastasis during the last 5 years (2014-2018). 


\section{Models by the Cancer Cell Inoculation Route}

The "seed and soil" theory by Paget is always used to describe the formation of bone metastases [21]. This theory explains the formation of metastases to preferred sites, and also creates the basis of using systemic cancer models. In systemic models, the cancer cells (seeds) are inoculated to the systemic circulation and let to home to their preferred sites (soils). These models are often described to be the real bone metastasis models as they include the early steps in the formation of metastases, including the survival in circulation, extravasation, dissemination to the bone, and finally dormancy and/or growth of bone metastases [1, 2, 4]. Additionally, disseminated and dormant tumor models have been characterized, bringing new possibilities to study these rare events $[1,2,3 \bullet]$. The systemic models have the disadvantages that they do not model tumor formation and intravasation from the primary tumor, and they do not allow the formation of pre-metastatic niches. Models depicting the full range from the primary tumor to overt metastases are scarce, and only a few models can be used to study the formation of metastases from the primary tumor. One such model is the 4T1 syngeneic breast cancer model $[3 \cdot, 4]$. In addition, for example, Pavese and colleagues published an orthotopic xenograft model resulting in prostate cancer cells in bone marrow but no detectable bone metastases [22]. A novel model was established by Valta and colleagues, that describes grafting renal cell cancer tissue slices orthotopically under the renal capsule resulting in bone metastases [23]. In systemic models, mostly used inoculation routes are intracardiac and, occasionally, intravenous routes. Intracardiac inoculation is used to reduce the number of lung metastases as inoculation to the left cardiac ventricle leads to peripheral blood circulation and uptake of tumor cells into bones before entering the lungs. In addition to these models, Kuchimaru and colleagues inoculated cancer cells to the caudal artery, leading to increased bone metastases with several cell lines, including human breast and prostate cancer cells [24]. This intracaudal model resulted in faster formation of bone metastases than with the intracardiac inoculation [24]. This model could be of high interest to many because the inoculation is relatively easy to perform and typically no mice are lost in the inoculations [24].

When considering large-scale efficacy studies with a specific interest in bone metastases, the disadvantage of using systemic models is that they often have a tumor take of 30-60\% in bone. The most commonly used breast cancer models, intracardiac inoculation of 4T1 or MDA-MB-231(SA) cells, make an exception with higher take rates. In addition to bone metastases, most mice do develop metastases also in soft tissues. Depending on the extent and location of the metastases, a subset of mice may be prematurely lost. If the primary interest is in bone, the use of intratibial or intrafemoral (also referred to as intraosseous) tumor models has some advantages [2, 4], including faster tumor formation, easier detection, more homogenous treatment groups, and decreased number of mice needed for the studies. Further improvement to the intratibial inoculation could be obtained by inoculation to the iliac artery, resulting in tumor growth in one hind limb, but avoiding the mechanical disruption of bone and ligaments [25]. The listing of established intratibial xenograft models is summarized by Jinnah [2] and Simmons [3•] and colleagues. LNCaP, LuCaP 35 and 23.1 [26], LuCaP 58 [27], LuCaP 136 [28], and $\mathrm{VCaP}$ $[29,30]$ intratibial prostate cancer models can be added to this listing. A summary of the models is listed in Table 1. Another aspect touching bone metastases is the possibility of secondary metastases. Formation of secondary metastases has been observed in two intratibial models of the $\mathrm{LuCaP}$ series, namely in LuCaP 58 resulting in liver and kidney metastases [27], and LuCaP 136 resulting in lung metastases [28].

\section{Models by Immunological Content}

Syngeneic models that can be used to study bone metastasis are listed by Jinnah [2] and Simmons [3•] and colleagues, and summarized in Table 1. Syngeneic models have mouse cancer cells inoculated into immunocompetent host [4]. Advantages of these models include rapid tumor formation and growth and, usually, a very high tumor take rate $[3 \cdot 4,4]$. Additionally, and more importantly, considering the current trend in oncology drug development, these models allow the species-specific interactions between the tumor and stromal cells, endocrine signaling, and immunological interactions [4]. However, the abovementioned issues can also represent disadvantages. The growth of mouse cancer cells is often regulated by other pathways than in human tumors [4]. For example, in the case of immune cell regulation of tumor growth, mouse tumor cells secrete different cytokines activating different immune cells than in humans, and also the mechanisms responsible for the formation of bone metastases have been speculated to be at least partially species-specific [4]. Hence, it is important to understand the differences and limitations of each model and the function and regulation of the therapeutic targets, and use multiple models in order to gain reliable and clinically predictive results from preclinical studies.

One approach to increased translation of preclinical findings could be achieved by using human immune system (HIS) engrafted mouse models. There is evidence that immune cells largely regulate tumor cell intravasation from the primary tumor, but also keep them dormant in the bone marrow, and for this reason clinically detectable bone metastases occur so late in humans [32]. In relation to bone metastasis and the immune system, Werner-Klein and colleagues successfully humanized immunodeficient mice using $\mathrm{CD} 34^{+}$hematopoietic progenitor cells isolated from bone marrow biopsies of nonmetastasizing breast, lung, prostate, or esophageal cancer 
Table 1 Summary of the breast, prostate, and lung cancer preclinical metastasis models described in the references sited in this review

\begin{tabular}{|c|c|c|c|}
\hline Category & $\begin{array}{l}\text { Immunological } \\
\text { content }\end{array}$ & Examples of the model & Reference \\
\hline \multirow[t]{6}{*}{$\begin{array}{l}\text { Systemic } \\
\text { models }\end{array}$} & $\begin{array}{c}\text { Syngeneic } \\
\text { models }\end{array}$ & Breast cancer: Met-1, 4T1 & {$[3 \cdot]$} \\
\hline & $\begin{array}{c}\text { Xenograft } \\
\text { models }\end{array}$ & $\begin{array}{l}\text { Breast cancer: MDA-MD-231, MDA-MB-435, } \\
\text { MCF-7, Zr-75-1, B02, } \\
\text { Prostate cancer: PC3, C4-2 }\end{array}$ & {$[2,3 \bullet]$} \\
\hline & & Stromal humanization: & \\
\hline & & Breast cancer: SUM1315, MDA-MB-231 & \\
\hline & & Prostate cancer: DU145, LNCaP, and PC3 & \\
\hline & & $\begin{array}{l}\text { Lung cancer: NCI-N417, NCI-H82, NCI-H446, } \\
\text { NCI-H146, NCI-H345, and NCI-H69 }\end{array}$ & \\
\hline \multirow[t]{6}{*}{$\begin{array}{l}\text { Local osseous } \\
\text { models }\end{array}$} & $\begin{array}{l}\text { Syngeneic } \\
\text { models }\end{array}$ & Breast cancer: Met-1 & {$[3 \cdot]$} \\
\hline & $\begin{array}{l}\text { Humanized } \\
\text { models }\end{array}$ & Breast cancer: BT-474, MDA-MB-231(SA) & {$[31]$} \\
\hline & $\begin{array}{c}\text { Xenograft } \\
\text { models }\end{array}$ & $\begin{array}{l}\text { Breast cancer: MDA-MD-231, MCF-7, BT-474 } \\
\text { Prostate cancer: PC3, LNCaP, Probasco, LuCaP } 35 \\
\text { and 23.1, LuCaP 58, LuCaP 136, VCaP }\end{array}$ & $\begin{array}{l}{[2,3 \bullet} \\
26-30]\end{array}$ \\
\hline & & $\begin{array}{l}\text { Lung cancer: SBC-5, SBC-3, SPC-A-1, A549, } \\
\text { H460, H727, NCI-H292, PC-9, PC-14, ACC-LC319, } \\
\text { LLC, H2030, and HARA }\end{array}$ & \\
\hline & & Stromal humanization: & \\
\hline & & Prostate cancer: DU145, LNCaP, and PC3 & \\
\hline
\end{tabular}

patients and provided a technique for this approach [18•]. The interactions between bone and immune cells are not yet fully established, but it is known that immune cells have a role in maintaining bone homeostasis [1, 2]. Since immune cells have been identified as important regulators of tumor growth and maintenance of bone homeostasis, they are most likely important contributors also in the context of bone metastasis. To study this, two breast cancer bone metastasis models were recently established in humanized mice using triple-positive BT-474 and triple-negative MDA-MB-231-SA human breast cancer cells with osteoblastic and osteolytic bone effects, respectively [31].

\section{Other Models Related to Bone Metastasis}

Other interesting approaches modeling bone metastasis can be divided into computational models, in vitro models, novel in vivo models, and cancer-induced bone pain models, which have started to gain wider interest. This review highlights the use of mice in preclinical studies, but it is important to also acknowledge the use of other species such as zebrafish as a model to study bone metastasis [33, 34].

A computational model for prostate cancer bone metastasis takes into consideration the number of osteoblast precursor cells; active osteoblasts and osteoclasts; normalized bone volume; early- and/or late-stage prostate cancer cells; boneregulatory proteins such as RANKL, osteoprotegerin (OPG), and PTHrP; WNT signaling; and prostate-specific antigen (PSA) [35]. The model divides bone metastases into high and low osteogenic stages correlating with high and low tumor burden in bone, resembling the clinical situation [35]. Other computational models include for example a model of cancer cell chemotaxis to bone [36].

Many in vitro approaches to study bone metastasis are summarized in a systematic review by Salamanna and colleagues [37] and by Krishnan and colleagues [38]. Marlow and colleagues have established an in vitro dormancy model with a co-culture of breast cancer cells and bone cells, and identified factors that keep cells in proliferatory or inhibitory (dormant) states [39]. Novel models combining in vivo to in vitro approaches include a bone-in-culture model by Wang and colleagues [40] where cancer cells are inoculated to the intra-iliac artery. After tumor is established, bones are cut into small pieces and placed in 96-well plates that can then be used for example for drug screening purposes [40]. In the same paper, the authors also showed correlation of drug responses in the established in vitro model and an in vivo bone metastasis model [40]. The novel use of the chorioallantoic membrane (CAM) model in the context of prostate cancer invasion and bone metastasis is also an interesting addition to the model portfolio [41]. In this assay, a bone chip is placed on top of the CAM and cancer cells are injected a small distance away from the bone, from where they can then invade and colonize the bone [41]. In many ways, this model settles in between the in vitro assays and mouse models. 
Many approaches are under investigations to improve clinical translation. One of the most interesting approaches is the "humanization" of mouse models [4, 18•]. Some of these models are described by Thibaudeau and colleagues [4]. These include for example humanization of the local microenvironment (for example mammary or prostate stroma) by creating transgenic mice, or also relating to this approach, inoculation of cancer cells with human stromal cells such as CAFs [4]. In the context of humanizing bone metastasis models, approaches have been taken to transplant the mice with human bone fragments and follow the migration of cancer cells from a different location to the bone fragment, and finally follow tumor growth in implanted human bone [2, 4]. Ectopic human bone can also be formed on site by implanting immature mesenchymal stromal cells from human bone marrow, or even blood-borne fibroblasts from umbilical cord blood $[42,43]$. The bone marrow space of the ossicle is colonized by mouse hematopoietic stem cells, but can also be transplanted with human $\mathrm{CD} 34^{+}$cells [44]. This approach has been useful in studying certain hematological malignancies that have been difficult to transplant [44]. Other novel humanized bone models utilize different kinds of bone scaffolds. A bone scaffold enables normal bone formation (humanized bone) in mice, and injection of breast cancer cells produced a bone metastasis model similar to metastasis in humans [45]. In a model established by Holzapfel and colleagues [46], mice are implanted with biodegradable tubular composite scaffolds together with mesenchymal progenitor cells synthetizing bone [46]. The implants in mice resemble normal bone structure with trabeculae and surrounding cortex [46]. In this model, prostate cancer cell migration into the bone and formation of metastasis were shown [46]. Hesami and colleagues have established a similar model where scaffolds seeded with human primary osteoblasts can be used to study the growth of human prostate cancer cells [47].

Cancer-induced bone pain models can be carried out in mice and rats [4]. These models are reviewed by Slosky and colleagues [48]. From these models, the Walker 256 breast cancer bone metastasis model and H1299 lung cancer bone metastasis model in rats can be used to monitor bone pain, which is one of the most common symptoms in bone metastatic patients $[49,50]$.

\section{Conclusions}

Cure for bone metastasis requires better understanding of the complex biology between tumor and bone. In addition, the immune system is likely to be a key player in the development and progression of bone metastases. As new treatments emerge, novel preclinical bone metastasis models that better recapitulate the situation in patients and concentrate on clinical predictivity should be established to shorten the gap in clinical translation and to improve patient survival in metastatic cancers.

\section{Compliance with Ethical Standards}

Conflict of Interest Tiina E. Kähkönen, Jenni Bernoulli, Jussi M. Halleen, and Mari I. Suominen are each an employee of Pharmatest Services.

Human and Animal Rights and Informed Consent This article does not contain any studies with human or animal subjects performed by any of the authors.

Open Access This article is distributed under the terms of the Creative Commons Attribution 4.0 International License (http:// creativecommons.org/licenses/by/4.0/), which permits unrestricted use, distribution, and reproduction in any medium, provided you give appropriate credit to the original author(s) and the source, provide a link to the Creative Commons license, and indicate if changes were made.

Publisher's Note Springer Nature remains neutral with regard to jurisdictional claims in published maps and institutional affiliations.

\section{References}

Papers of particular interest, published recently, have been highlighted as:

- Of importance

•. Of major importance

1. Johnson RW, Suva LJ. Hallmarks of bone metastasis. Calcif Tissue Int. 2018;102(2):141-51.

2. Jinnah AH, Zacks BC, Gwam CU, Kerr BA. Emerging and established models of bone metastasis. Cancers (Basel). 2018;10(6):E176. https://doi.org/10.3390/cancers10060176.

3. Simmons JK, Hildreth BE 3rd, Supsavhad W, Elshafae SM, Hassan BB, Dirksen WP, et al. Animal models of bone metastasis. Vet Pathol. 2015;52(5):827-41 Review article describing the bone metastasis models by cancer type both in syngeneic and xenograft setting, as well as some transgenic mouse models, with a highlight on imaging of bone metastasis in vivo.

4. Thibaudeau L, Quent VM, Holzapfel BM, Taubenberger AV, Straub M, Hutmacher DW. Mimicking breast cancer-induced bone metastasis in vivo: current transplantation models and advanced humanized strategies. Cancer Metastasis Rev. 2014;33(2-3):72135 .

5. Ren G, Esposito M, Kang Y. Bone metastasis and the metastatic niche. J Mol Med (Berl). 2015;93(11):1203-12 A very comprehensive review on the bone metastatic process.

6. Giles AJ, Reid CM, Evans JD, Murgai M, Vicioso Y, Highfill SL, et al. Activation of hematopoietic stem/progenitor cells promotes immunosuppression within the pre-metastatic niche. Cancer Res. 2016;76(6):1335-47.

7. Kelly T, Suva LJ, Huang Y, Macleod V, Miao HQ, Walker RC, et al. Expression of heparanase by primary breast tumors promotes bone resorption in the absence of detectable bone metastases. Cancer Res. 2005;65(13):5778-84.

8. Peinado H, Aleckovic M, Lavotshkin S, Matei I, Costa-Silva B, Moreno-Bueno G, et al. Melanoma exosomes educate bone marrow 
progenitor cells toward a pro-metastatic phenotype through MET. Nat Med. 2012;18(6):883-91.

9. Valencia K, Luis-Ravelo D, Bovy N, Anton I, Martinez-Canarias S, Zandueta $\mathrm{C}$, et al. miRNA cargo within exosome-like vesicle transfer influences metastatic bone colonization. Mol Oncol. 2014;8(3): 689-703.

10.• Barcellos-de-Souza P, Comito G, Pons-Segura C, Taddei ML, Gori $\mathrm{V}$, Becherucci V, et al. Mesenchymal stem cells are recruited and activated into carcinoma-associated fibroblasts by prostate cancer microenvironment-derived TGF-beta1. Stem Cells. 2016;34(10): 2536-47 This work emphasizes the importance on bone marrow-derived cells in cancer progression.

11. Giannoni E, Bianchini F, Calorini L, Chiarugi P. Cancer associated fibroblasts exploit reactive oxygen species through a proinflammatory signature leading to epithelial mesenchymal transition and stemness. Antioxid Redox Signal. 2011;14(12):2361-71.

12. Krishnan V, Vogler EA, Sosnoski DM, Mastro AM. In vitro mimics of bone remodeling and the vicious cycle of cancer in bone. J Cell Physiol. 2014;229(4):453-62.

13. Mountzios G, Terpos E, Syrigos K, Papadimitriou C, Papadopoulos G, Bamias A, et al. Markers of bone remodeling and skeletal morbidity in patients with solid tumors metastatic to the skeleton receiving the biphosphonate zoledronic acid. Transl Res. 2010;155(5):247-55.

14. Lim W, Sohn H, Ko Y, Park M, Kim B, Jo D, et al. Real-time in vivo imaging of metastatic bone tumors with a targeted nearinfrared fluorophore. Oncotarget. 2017;8(39):65770-7.

15. Lee SH, Park SA, Zou Y, Seo SU, Jun CD, Lee WJ, et al. Real-time monitoring of cancer cells in live mouse bone marrow. Front Immunol. 2018;9:1681 This paper describes an important advancement enabling following of disseminated tumor cells in the bone marrow.

16. Ferreira A, Alho I, Casimiro S, Costa L. Bone remodeling markers and bone metastases: from cancer research to clinical implications. Bonekey Rep. 2015;4:668.

17. Martin-Fernandez M, Valencia K, Zandueta C, Ormazabal C, Martinez-Canarias S, Lecanda F, et al. The usefulness of bone biomarkers for monitoring treatment disease: a comparative study in osteolytic and osteosclerotic bone metastasis models. Transl Oncol. 2017;10(2):255-61.

18. Morton JJ, Bird G, Refaeli Y, Jimeno A. Humanized mouse xenograft models: narrowing the tumor-microenvironment gap. Cancer Res. 2016;76(21):6153-8 This review presents many important aspects of the use of mice with humanized immune system in oncology studies.

19. Rampetsreiter P, Casanova E, Eferl R. Genetically modified mouse models of cancer invasion and metastasis. Drug Discov Today Dis Model. 2011;8(2-3):67-74.

20. Gingrich JR, Barrios RJ, Morton RA, Boyce BF, DeMayo FJ, Finegold MJ, et al. Metastatic prostate cancer in a transgenic mouse. Cancer Res. 1996;56(18):4096-102.

21. Paget S. The distribution of secondary growths in cancer of the breast. 1889. Cancer Metastasis Rev. 1989;8(2):98-101.

22. Pavese J, Ogden IM, Bergan RC. An orthotopic murine model of human prostate cancer metastasis. J Vis Exp. 2013;(79):e50873. https://doi.org/10.3791/50873.

23. Valta MP, Zhao H, Ingels A, Thong AE, Nolley R, Saar M, et al. Development of a realistic in vivo bone metastasis model of human renal cell carcinoma. Clin Exp Metastasis. 2014;31(5):573-84.

24. Kuchimaru T, Kataoka N, Nakagawa K, Isozaki T, Miyabara H, Minegishi M, et al. A reliable murine model of bone metastasis by injecting cancer cells through caudal arteries. Nat Commun. 2018;9(1):2981 018-05366-3.

25. Yu C, Wang H, Muscarella A, Goldstein A, Zeng HC, Bae Y, et al. Intra-iliac artery injection for efficient and selective modeling of microscopic bone metastasis. J Vis Exp. 2016;(115). https://doi. org/10.3791/53982.

26. Corey E, Quinn JE, Bladou F, Brown LG, Roudier MP, Brown JM, et al. Establishment and characterization of osseous prostate cancer models: intra-tibial injection of human prostate cancer cells. Prostate. 2002;52(1):20-33.

27. Suominen MI, Fagerlund KM, Rissanen JP, Konkol YM, Morko JP, Peng Z, et al. Radium-223 inhibits osseous prostate cancer growth by dual targeting of cancer cells and bone microenvironment in mouse models. Clin Cancer Res. 2017;23(15):4335-46.

28. Valta MP, Zhao H, Saar M, Tuomela J, Nolley R, Linxweiler J, et al. Spheroid culture of LuCaP 136 patient-derived xenograft enables versatile preclinical models of prostate cancer. Clin Exp Metastasis. 2016;33(4):325-37.

29. Kähkönen TE, Suominen MI, Halleen JM, Bernoulli J, Lejeune P. Castration-resistant prostate cancer bone metastasis model to assess new therapeutics. Cancer Res. 2018;78(13Supplement):1162.

30. Graham TJ, Box G, Tunariu N, Crespo M, Spinks TJ, Miranda S, et al. Preclinical evaluation of imaging biomarkers for prostate cancer bone metastasis and response to cabozantinib. J Natl Cancer Inst. 2014;106(4):dju033.

31. Kähkönen TE, Suominen MI, Halleen JM, Haapaniemi T, Tanaka A, Seiler M, et al. Humanized mouse models of triple-negative and triple-positive breast cancer for preclinical validation of novel immuno-oncology therapies. Eur J Cancer. 2018;92:S7-8.

32. Baschuk N, Rautela J, Parker BS. Bone specific immunity and its impact on metastasis. Bonekey Rep. 2015;4:665.

33. Brown HK, Schiavone K, Tazzyman S, Heymann D, Chico TJ. Zebrafish xenograft models of cancer and metastasis for drug discovery. Expert Opin Drug Discov. 2017;12(4):379-89.

34. Chen L, Groenewoud A, Tulotta C, Zoni E, Kruithof-de Julio M, van der Horst G, et al. A zebrafish xenograft model for studying human cancer stem cells in distant metastasis and therapy response. Methods Cell Biol. 2017;138:471-96.

35. Farhat A, Jiang D, Cui D, Keller ET, Jackson TL. An integrative model of prostate cancer interaction with the bone microenvironment. Math Biosci. 2017;294:1-14.

36. Munoz AI, Tello JI. On a mathematical model of bone marrow metastatic niche. Math Biosci Eng. 2017;14(1):289-304.

37. Salamanna F, Contartese D, Maglio M, Fini M. A systematic review on in vitro 3D bone metastases models: a new horizon to recapitulate the native clinical scenario? Oncotarget. 2016;7(28):44803-20.

38. Krishnan V, Vogler EA, Mastro AM. Three-dimensional in vitro model to study osteobiology and osteopathology. J Cell Biochem. 2015;116(12):2715-23.

39. Marlow R, Honeth G, Lombardi S, Cariati M, Hessey S, Pipili A, et al. A novel model of dormancy for bone metastatic breast cancer cells. Cancer Res. 2013;73(23):6886-99.

40. Wang H, Tian L, Goldstein A, Liu J, Lo HC, Sheng K, et al. Bonein-culture array as a platform to model early-stage bone metastases and discover anti-metastasis therapies. Nat Commun. 2017;8: 15045 .

41. San Martin R, Pathak R, Jain A, Jung SY, Hilsenbeck SG, PinaBarba $\mathrm{MC}$, et al. Tenascin-C and integrin alpha9 mediate interactions of prostate cancer with the bone microenvironment. Cancer Res. 2017;77(21):5977-88.

42. Pettway GJ, McCauley LK. Ossicle and vossicle implant model systems. Methods Mol Biol. 2008;455:101-10.

43. Pievani A, Sacchetti B, Corsi A, Rambaldi B, Donsante S, Scagliotti V, et al. Human umbilical cord blood-borne fibroblasts contain marrow niche precursors that form a bone/marrow organoid in vivo. Development. 2017;144(6):1035-44.

44. Reinisch A, Thomas D, Corces MR, Zhang X, Gratzinger D, Hong WJ, et al. A humanized bone marrow ossicle xenotransplantation model enables improved engraftment of healthy and leukemic human hematopoietic cells. Nat Med. 2016;22(7):812-21. 
45. Quent V, Taubenberger AV, Reichert JC, Martine LC, Clements JA, Hutmacher DW, et al. A humanised tissue-engineered bone model allows species-specific breast cancer-related bone metastasis in vivo. J Tissue Eng Regen Med. 2018;12(2):494-504.

46. Holzapfel BM, Wagner F, Loessner D, Holzapfel NP, Thibaudeau L, Crawford R, et al. Species-specific homing mechanisms of human prostate cancer metastasis in tissue engineered bone. Biomaterials. 2014;35(13):4108-15.

47. Hesami P, Holzapfel BM, Taubenberger A, Roudier M, Fazli L, Sieh $\mathrm{S}$, et al. A humanized tissue-engineered in vivo model to dissect interactions between human prostate cancer cells and human bone. Clin Exp Metastasis. 2014;31(4):435-46.
48. Slosky LM, Largent-Milnes TM, Vanderah TW. Use of animal models in understanding cancer-induced bone pain. Cancer Growth Metastasis. 2015;8(Suppl 1):47-62.

49. Shenoy PA, Kuo A, Vetter I, Smith MT. The Walker 256 breast cancer cell-induced bone pain model in rats. Front Pharmacol. 2016;7:286.

50. Wang G, Chen J, Ma R, Xu W, Yan C, Niu C. Effects of zoledronic acid and ibandronate in the treatment of cancer pain in rats with lung cancer combined with bone metastases. Oncol Lett. 2018;16(2):1696-700. 